BEATA SKRZYDLEWSKA - LUBLIN

\title{
DIE KIRCHLICHEN MUSEEN ZUM 100. JAHRESTAG IHRES WIRKENS. ZUSAMMENFASSUNG DER KONFERENZ
}

Am 26. September 2005 fand eine vom Zentrum für Kirchliche Archive, Bibliotheken und Museen (ABMK) in Zusammenarbeit mit dem Lehrstuhl für Museumswesen und Denkmalschutz im Institut für Kunstgeschichte der Katholischen Universität Lublin (KUL) organisierte Konferenz statt.

Der Leitgedanke der Beratungen stand im Zusammenhang mit der Tatsache, daß viele Museen in den letzten Jahren ihr einhundertjähriges Bestehen begingen oder dieses demnächst begehen werden. Die Thematik der Referate konzentrierte sich auf die wichtigsten Fragen im Zusammenhang mit dem kirchlichen Museumswesen.

Die Teilnehmer wurden begrüßt vom Prorektor der KUL, Prof. Dr. habil. Józef Fert, und die offizielle Eröffnung vollzog P. Prof. Dr. habil. Marek Zahajkiewicz, der Direktor des ABMK-Zentrums.

Die Beratungen wurden in drei Themengruppen gegliedert:

\section{Die Geschichte der kirchlichen Museen und ihre Rechtsgrundlagen}

Diesen Teil eröffnete Dr. Beata Skrzydlewska, Mitarbeiterin am Lehrstuhl für Museumswesen und Denkmalschutz im Institut für Kunstgeschichte der KUL. Sie hielt ein Referat zum Thema "Der Stand der Forschungen zum kirchlichen Museumswesen in Polen" und machte darauf aufmerksam, daß die mit dem kirchlichen Museumswesen verbundenen einzelnen Fragen in Publikationen aus dem Bereich der Geschichte, der Kunstgeschichte, des Museumswesens und in populärwissenschaftlichen Veröffentlichungen erörtert werden. Obwohl es kirchliche Museen schon seit der ersten Hälfte des 19. Jahrhunderts gibt, ist bisher noch keine Abhandlung entstanden, die ihre Geschichte systematisch und umfassend analysiert und die Spezifik dieser Einrichtungen behandelt. 
P. Magister Henryk Pyka, der Direktor des Diözesanmuseums in Katowice, präsentierte in seinem Referat "Die Geschichte der Diözesanmuseen von ihrer Entstehung bis zur Gegenwart" die Geschichte der kirchlichen Museen in Polen, angefangen von den ältesten wie den Museen in Włocławek, Płock oder Sandomierz bis hin zu dem neuesten, im Jahre 2004 gegründeten Museum in Drohiczyn.

Bischof Dr. Mariusz Leszczyński, der Direktor des Diözesanmuseums in Zamość und Herausgeber des "Biuletyn Kościelnych Dóbr Kultury" ("Bulletin für Kirchliche Kulturgüter"), besprach in seinem Referat "Die kirchlichen Museen in der Legislatur der Kirche" die neuesten vom Heiligen Stuhl herausgegebenen Dokumente. Besonders verwies er auf das "Rundschreiben" der Päpstlichen Kommission für die Kulturgüter der Kirche "Die pastorale Funktion der kirchlichen Archive".

Der langjährige Direktor des Diözesanmuseums in Tarnów P. Dr. Władysław Szczebak sprach zum Thema "Die Pfarr- und Klostermuseen am Beispiel der Diözese Tarnów". In dieser Diözese - wie in nur wenigen in Polen - fungieren die Pfarr- und Klostermuseen als Zweigstellen des Diözesanmuseums.

P. Magister Andrzej Rusak vom Diözesanmuseum in Sandomierz erinnerte in seinem Referat "Die Museumsräume und die in ihnen herrschenden Bedingungen zur Aufbewahrung der Bestände" an die Notwendigkeit einer entsprechenden Sicherung der Objekte, sowohl in den Lagerräumen als auch unter Austellungsbedingungen.

\section{Die Tätigkeit der kirchlichen Museen}

Dieser Teil der Beratungen war den wichtigsten Aufgaben der kirchlichen Museen gewidmet.

Als erster sprach der Direktor des Archidiözesanmuseums in Poznań, P. Magister Marian Lewandowski. In seinem Referat "Die Inventarisierung der Musealien" verwies er auf die Methoden und sich verändernden Arten der Katalogisierung und Aufbereitung der Objekte in den letzten mehr als hundert Jahren.

P. Magister Paweł Tkaczyk, der Direktor des neu entstandenen Diözesanmuseums in Kielce, befaßte sich in seinem Referat "Die Betreuung der Kunstwerke vor Ort" mit der Frage der Betreuung der Denkmäler vor Ort durch die Mitarbeiter der kirchlichen Museen, und P. Magister Wojciech Szlachetka aus Lublin machte in seinem Vortrag "Die Konservation der Bestände in den Museen" auf die großen Möglichkeiten aufmerksam, die die konservatorischen Werkstätten der kirchlichen Museen bieten. Diese Einrichtungen garantieren nicht nur den Schutz der Museumsbestände, sondern in ihnen können auch Kunstwerke aus dem Gebiet der Diözese gerettet werden.

\section{Die kulturelle Funktion der kirchlichen Museen}

Im dritten Teil referiereten: Prof. Dr. habil. Urszula Mazurczak, die Kuratorin des Lehrstuhls für Museumswesen und Denkmalschutz im Institut für Kunstge- 
schichte der KUL, die zum Thema "Die Früchte des kirchlichen Museumswesens im Bereich der Wissenschaft" sprach, P. Magister Stefan Cegłowski, der langjährige Kustode des Diözesanmuseums in Płock, der über "Die kulturschaffende Rolle des Diözesanmuseums in der kirchlichen Gemeinschaft" referierte, und Dr. Beata Purc-Stępniak, deren Referat lautete: "Das Nationalmuseum in Gdańsk seine Entstehungsgeschichte und seine Funktion in der Struktur und Kultur der Stadt".

In der Diskussion wurde auf die dringende Notwendigkeit einer engeren $\mathrm{Zu}$ sammenarbeit der kirchlichen Museen mit den akademischen Zentren verwiesen, insbesondere mit der Katholischen Universität Lublin "Johannes Paul II.". Eine solche Zusammenarbeit gibt es schon seit einer Reihe von Jahren, unter anderem durch die vom ABMK-Zentrum seit 1959 zyklisch organisierten Tagungen zum kirchlichen Museumswesen sowie durch die Beteiligung des Instituts für Kunstgeschichte der KUL an den Museumsarbeiten. Regelmäßig finden dreiwöchige Museumspraktika für Studenten des 4. Studienjahres statt, in denen die Studenten Dokumentationsarbeiten in den Museen ausführen. In den letzten Jahren nahmen sie an den Arbeiten der Diözesanmuseen in Przemyśl und in Sandomierz teil und halfen bei der Inventarisierung der Bestände des Museums für Missionsgeschichte der Missionarspriester in Kraków. Zur Ausweitung dieser Zusammenarbeit bietet sich eine besondere Gelegenheit, denn im Institut für Kunstgeschichte der KUL gibt es seit September 2005 einen Lehrstuhl für Museumswesen und Denkmalschutz. Mit dieser Hilfe werden die Einrichtungen ihre Probleme mit der Finanzierung der Instandhaltung beweglicher Denkmäler, der Schaffung einer zentralen Datenbank für kirchliche Museumsobjekte an der KUL oder der Organisierung von Weiterbildungskursen für Geistliche auf dem Gebiet des Museumswesens und Denkmalschutzes lösen können. 